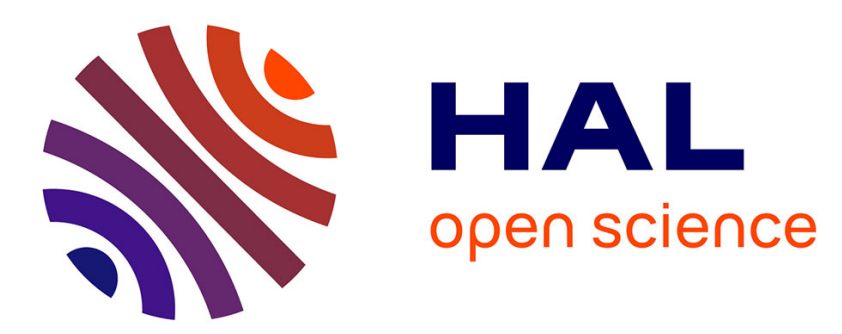

\title{
Controllability of one-dimensional viscous free boundary flows
}

Borjan Geshkovski, Enrique Zuazua

\section{To cite this version:}

Borjan Geshkovski, Enrique Zuazua. Controllability of one-dimensional viscous free boundary flows. 2021. hal-02277740v2

\section{HAL Id: hal-02277740 \\ https://hal.science/hal-02277740v2}

Preprint submitted on 11 Mar 2021

HAL is a multi-disciplinary open access archive for the deposit and dissemination of scientific research documents, whether they are published or not. The documents may come from teaching and research institutions in France or abroad, or from public or private research centers.
L'archive ouverte pluridisciplinaire HAL, est destinée au dépôt et à la diffusion de documents scientifiques de niveau recherche, publiés ou non, émanant des établissements d'enseignement et de recherche français ou étrangers, des laboratoires publics ou privés. 


\title{
CONTROLLABILITY OF ONE-DIMENSIONAL VISCOUS FREE BOUNDARY FLOWS
}

\author{
BORJAN GESHKOVSKI AND ENRIQUE ZUAZUA
}

\begin{abstract}
In this work, we address the local controllability of a one-dimensional free boundary problem for a fluid governed by the viscous Burgers equation. The free boundary manifests itself as one moving end of the interval, and its evolution is given by the value of the fluid velocity at this endpoint. We prove that, by means of a control actuating along the fixed boundary, we may steer the fluid to constant velocity in addition to prescribing the free boundary's position, provided the initial velocities and interface positions are close enough.
\end{abstract}

\section{CONTENTS}

1. Introduction and main result 1

2. Reformulation of the problem 6

3. Null-controllability of the linearized system 8

4. The nonlinear problem 15

5. Concluding remarks $\quad 18$

References $\quad 20$

Keywords. Controllability, free boundary problem, viscous Burgers equation.

AMS Subject Classification 93B05, 35R35, 35Q35, 93C20.

\section{INTRODUCTION AND MAIN RESULT}

Let $T>0$ be a given positive time. We consider the following problem for the viscous Burgers equation:

$$
\begin{cases}v_{t}-v_{z z}+v v_{z}=0 & \text { in }(0, T) \times(0, \ell(t)) \\ v(t, 0)=u(t), \quad v_{z}(t, \ell(t))=0 & \text { in }(0, T) \\ \ell^{\prime}(t)=v(t, \ell(t)) & \text { in }(0, T) \\ v(0, z)=v_{0}(z), \quad \ell(0)=\ell_{0} & \text { in }\left(0, \ell_{0}\right) .\end{cases}
$$

System (1.1) is a free boundary problem, where the unknown is the pair $(v, \ell)$, with $\ell$ representing the free boundary. Here $\ell_{0}>0$, and $u=u(t)$ is a control actuating along the fixed boundary $z=0$. Henceforth and in the above, we use the notation $(0, T) \times(0, \ell(t))$ for the set $\{(t, z) \in(0, T) \times \mathbb{R}: 0<z<\ell(t)\}$, with analogue notation for the closure of the latter.

Model (1.1) is presented and studied by Caboussat \& Rappaz in [5, 6], where local-intime existence and uniqueness of strong solutions are shown, supplemented by numerical studies. It may be seen as a one-dimensional simplification of the incompressible

Date: February 19, 2021. 
Navier-Stokes equations with a free surface set in $\mathbb{R}^{d}$ with $d=2,3$, as encountered in the works of Beale [2, 3], and Maronnier, Picasso \& Rappaz [29], where particular emphasis is given on the application to mould filling. The state of System (1.1) involves the velocity $v(t, z)$ of the one-dimensional fluid and the free boundary $\ell(t)$, whose counterpart in dimension $d \geqslant 2$ would represent the position of the free surface of the fluid. The fluid velocity is governed by the viscous Burgers equation, while the dynamics of the free boundary follow the fluid velocity, as per the equation $\ell^{\prime}(t)=v(t, \ell(t))$.

As the state of the system (1.1) consists of two components $(v, \ell)$, the natural exactcontrollability problem, which is the main goal of this work, is to steer both components to a priori defined targets in a given time $T>0$. This would ensure the entire system remains in such a configuration after the time $T$ has elapsed. Formulated as such, this control problem has not been accurately addressed in the literature for systems where the coupling between the PDE and ODE components is only done through the boundary of the domain, as in (1.1). Through this work, we aim to present a systematic and ordered methodology for addressing such compound control problems.

The most general and feasible targets to which one may control both components of (1.1) are time-dependent trajectories of (1.1), namely free solutions to (1.1). The question of controllability to non-trivial trajectories is however not straightforward at all. This is observed on the level of the system linearized around the non-trivial target trajectory, which contains several non-local trace terms (see (5.1)). Consequently, in terms of the adjoint problem one obtains non-standard boundary conditions (see (5.3)) for which, up to the best of our knowledge, observability inequalities are lacking. This is discussed in more detail in Section 5.1, and the general problem of controllability to arbitrary trajectories remains open.

At this point, we observe that for any $\ell_{*}>0$, the pair $(\bar{v}, \bar{\ell})$ with

$$
\bar{v} \in \mathbb{R}, \quad \bar{\ell}(t)=\ell_{*}+\bar{v} t>0 \quad \text { in }[0, T],
$$

is an explicit, non-trivial solution to System (1.1) with $u \equiv \bar{v}$. As discussed in Section 2, the system linearized around this trajectory does not manifest the issues appearing in the general trajectory case. The main goal of this work is to prove the local exactcontrollability for (1.1) to this particular trajectory. To be more precise, given an arbitrary constant velocity $\bar{v}$ and an initial position $\ell_{*}$, we want to show that whenever $\left(v_{0}, \ell_{0}\right)$ are sufficiently close to $\left(\bar{v}, \ell_{*}\right)$ (see Figure 1 ), one can find a control $u(t)$ such that the corresponding trajectory $(v, \ell)$ to $(1.1)$ connects $\left(v_{0}, \ell_{0}\right)$ to the target $\left(\bar{v}, \ell_{*}+\right.$ $\bar{v} T$ ) at time $T$. This is reflected in our main result.

Theorem 1.1. Let $T>0, \ell_{*}>0$ and $\bar{v} \in \mathbb{R}$ be such that $\bar{\ell}(t)=\ell_{*}+\bar{v} t>0$ for all $t \in[0, T]$. There exists $r>0$ such that for all $\ell_{0}>0$ and $v_{0} \in H^{1}\left(0, \ell_{0}\right)$ satisfying

$$
\left\|v_{0}-\bar{v}\right\|_{H^{1}\left(0, \ell_{0}\right)}+\left|\ell_{0}-\ell_{*}\right| \leqslant r
$$

there exists a control $u \in H^{3 / 4}(0, T)$ such that the unique solution

$$
\ell \in C^{1}([0, T]) \quad v \in L^{2}\left(0, T ; H^{2}(0, \ell(\cdot))\right) \cap C^{0}\left([0, T] ; H^{1}(0, \ell(\cdot))\right)
$$

of (1.1) satisfies

$$
\inf _{t \in[0, T]} \ell(t)>0 \quad \text { and } \quad \ell(T)=\bar{\ell}(T) \quad \text { and } \quad v(T, \cdot)=\bar{v} \quad \text { in }(0, \ell(T)) .
$$


Moreover, one has

$$
\|u\|_{H^{3 / 4}(0, T)} \lesssim_{T}\left\|v_{0}-\bar{v}\right\|_{H^{1}\left(0, \ell_{0}\right)}+\left|\ell_{0}-\ell_{*}\right| .
$$

Our proof combines several elements of control of parabolic equations in a systematic and ordered way, in view of establishing a well-defined and clear methodology for tackling controllability problems for free boundary systems such as (1.1).

A couple of remarks are in order.

Remark 1.1. It is readily seen that Theorem 1.1 also covers the case of null-controllability of the state and prescribing the position of the interface, by considering $(\bar{v}, \bar{\ell})=\left(0, \ell_{*}\right)$ with $\ell_{*}>0$. Aside from the trivial solution $\left(0, \ell_{*}\right)$, we may also look to potentially control to the stationary solutions of (1.1), namely, time-independent solutions. In other words, given $\ell_{*}>0$ and $\bar{v} \in \mathbb{R}$ we seek to compute the solutions to

$$
\begin{cases}-v_{z z}+v v_{z}=0 & \text { in }\left(0, \ell_{*}\right) \\ v(0)=\bar{v}, \quad v\left(\ell_{*}\right)=0, \quad v_{z}\left(\ell_{*}\right)=0 . & \end{cases}
$$

It may be checked that the only solution to the second-order equation in (1.3) is $v \equiv 0$, which enhances our interest in time-dependent trajectories as targets.

Remark 1.2. The result we prove here is local (a global result is not known also for similar problems such as (1.4), (1.5)). One may think of combining this local result with a stabilization argument, which, should stabilization hold, would allow to steer System (1.1) to a neighborhood of the target wherein the local controllability result applies. However, while the PDE component may possess an inherent dissipative mechanism, the asymptotic position of the free boundary is generally not known for problems of this nature. See Section 5.2 for more details.

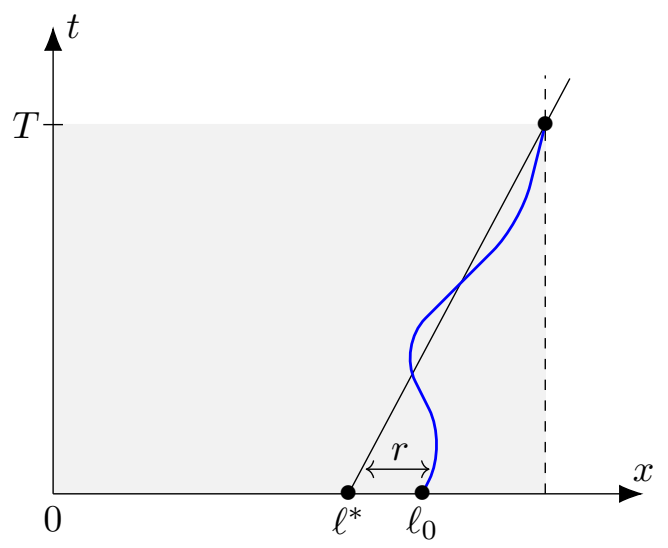

Figure 1. Controllability of the position of the free surface $\ell$ (blue curve) to the reference interface $\bar{\ell}$ (black) at time $T$, provided the initial positions are close enough. 
1.1. State of the art. The controllability aspects of one-dimensional, parabolic freeboundary problems similar to (1.1) have been addressed in several recent works (see e.g. $[13,17,16,19])$. In $[13,17]$, Fernández-Cara et al. consider the one-phase Stefan problem

$$
\begin{cases}v_{t}-v_{z z}=0 & \text { in }(0, T) \times(0, \ell(t)) \\ v(t, 0)=u(t), \quad v(t, \ell(t))=0 & \text { in }(0, T) \\ \ell^{\prime}(t)=-v_{z}(t, \ell(t)) & \text { in }(0, T) \\ v(0, z)=v_{0}(z), \quad \ell(0)=\ell_{0} & \text { in }\left(0, \ell_{0}\right) .\end{cases}
$$

We stress that in [13,17], a null-controllability result where only the first component $v$ is controlled is shown, i.e. $v(T, \cdot)=0$ in $(0, \ell(T))$, for small initial data $v_{0}$. Such results are partial as they cannot ensure that the entire system remain in such the prescribed configuration past the time horizon $T$. The authors' proof relies on fixing the free boundary $\ell \in C^{1}([0, T])$ (and removing the equation for the velocity $\ell^{\prime}$ ), and proving an observability inequality for the linear heat equation in the non-cylindrical domain $(0, T) \times(0, \ell(t))$, with a constant uniform in $\ell$. The conclusion for (1.4) follows by means of a Schauder fixed-point argument applied to the map $\ell \longmapsto \ell_{0}-\int_{0} v_{x}^{\ell}(\tau, \ell(\tau)) \mathrm{d} \tau$ in an appropriate subspace of $C^{1}([0, T])$. In [16], the authors obtain the same local controllability result by means of a different technique, which relies on a transformation to a fixed domain, a linear controllability test and an inverse function argument. Our strategy of proof for the controllability of both states of (1.1) has some resemblance to that in [16], but with several important technical differences. Moreover, with small adjustments, the control strategy we present herein also yields a local null-controllability result for both the solution and the free boundary of the Stefan problem (1.4), namely $\ell(T)=\ell_{*}$ and $v(T, \cdot)=0$ in $\left(0, \ell_{*}\right)$ whenever $v_{0}$ and $\ell_{0}-\ell_{*}$ are small enough.

1.1.1. Comparison with fluid-structure interaction problems. Free boundary problems which arise in fluid-structure interaction have also been addressed. Doubova \& FernándezCara [14] as well as Liu, Takahashi \& Tucsnak [26] consider the system

$$
\begin{cases}v_{t}-v_{z z}+v v_{z}=0 & \text { in }(0, T) \times(-1, \ell(t)) \cup(\ell(t), 1) \\ v(t,-1)=u_{1}(t), \quad v(t, 1)=u_{2}(t) & \text { in }(0, T) \\ v(t, \ell(t))=\ell^{\prime}(t) & \text { in }(0, T) \\ m \ell^{\prime \prime}(t)=\left[v_{z}\right](t, \ell(t)) & \text { in }(0, T) \\ v(0, z)=v_{0}(z), \quad \ell(0)=\ell_{0}, \quad \ell^{\prime}(0)=\ell_{1} & \text { in }\left(-1, \ell_{0}\right) \cup\left(\ell_{0}, 1\right),\end{cases}
$$

which is first introduced by Vázquez \& Zuazua [33, 34], where global in-time wellposedness, self-similar asymptotics and particle collision are addressed (see also [27] for a related study). The free boundary $\ell(t)$ represents the displacement/position of a solid particle of mass $m>0$, which splits the domain in two parts. The null-controllability of (1.5) refers to controlling three components: the fluid velocity $v(T, \cdot)=0$, the particle velocity $\ell^{\prime}(T)=0$, and the particle's position $\ell(T)=0$.

In [14], controls $u_{1}, u_{2}$ are used on both boundaries in view of applying a Carleman based strategy. Such an approach is not feasible when there is a control at only one end (i.e. $u_{2}=0$ ) because of the lack of connectivity of the fluid domain. This issue was mended in [26], where the authors introduce a systematic methodology for tackling the null-controllability of parabolic systems in spite of source terms, without requiring Carleman inequalities (they thus use spectral techniques). We also refer to 
the work of Cindea, Micu, Roventa and Tucsnak [7], where the authors consider a control actuating only on the moving particle: $m \ell^{\prime \prime}(t)=\left[v_{z}\right](t, \ell(t))+u(t)$. They prove global null-controllability (in large time) for the fluid and particle velocities, and approximate controllability for the particle's position. We refer to the recent work [32] for a technical improvement of this result. The lack of connectivity of the fluid domain does not appear in two and three dimensions, and the Carleman-based approach has been successfully applied for proving local null-controllability results for fluid-rigidbody systems (see $[4,20]$ and the references therein) where the control is generally actuating along a part of the fixed boundary.

Remark 1.3. At this point we remark that there is a notable difference between problems of the type (1.5) and (1.1). Indeed, the former system has a stronger coupling than the latter systems due to the presence of two equations for the free boundary $\ell$. This can be seen when linearizing both systems around their trivial trajectory (after fixing the domain). In the linearization of (1.1) (see (5.1) with $a \equiv 1, b, c, d, e \equiv 0$ and Section 2 for details),

$$
\begin{cases}y_{t}-y_{x x}=0 & \text { in }(0, T) \times(0,1) \\ y(t, 0)=u(t), \quad y_{x}(t, 1)=0 & \text { in }(0, T) \\ \ell^{\prime}(t)=y(t, 1) & \text { in }(0, T) \\ y(0, x)=y_{0}(x), \quad \ell(0)=\ell_{0} & \text { in }(0,1),\end{cases}
$$

the PDE and ODE components are decoupled, as the linear PDE may be solved without any knowledge of the ODE component. On the other hand, the linearization of (1.5) around the trivial solution (see [26])

$$
\begin{cases}y_{t}-y_{x x}=0 & \text { in }(0, T) \times(-1,0) \cup(0,1) \\ y(t,-1)=u(t), \quad y(t, 1)=0 & \text { in }(0, T) \\ y(t, 0)=\ell^{\prime}(t) & \text { in }(0, T) \\ m \ell^{\prime \prime}(t)=\left[y_{x}\right](t, 0) & \text { in }(0, T) \\ y(0, x)=y_{0}(x), \quad \ell(0)=\ell_{0}, \quad \ell^{\prime}(0)=\ell_{1} & \text { in }\left(-1, \ell_{0}\right) \cup\left(\ell_{0}, 1\right),\end{cases}
$$

preserves the coupling of the PDE component and the ODE component because of the presence of two equations for the latter.

In the above-cited works on fluid-structure problems, the controllability problem addressed is that of controlling the PDE component to zero and the ODE component(s) to some given reference points. For the case of non-trivial stationary solutions and trajectories as targets, much less is known. In [1], Badra \& Takahashi prove feedback stabilization to non-trivial stationary solutions for (1.5). Therein, it can also be seen that the question of controllability to non-trivial stationary solutions is not straightforward. This is observed on the level of the system linearized around the target, which contains several trace terms (as in (5.1)). As a result, in terms of the adjoint problem, one obtains non-local boundary conditions (similar to (5.3)), for which observability inequalities are lacking.

We also refer to Dunbar et al. [10,9] for motion planning and flatness control, and Krstic et al. [21, 22, 23, 24] and the references therein for feedback stabilization via backstepping design of the Stefan problem (1.4), see also Phan \& Rodrigues [31] for stabilization to trajectories for general parabolic problems. 
As discussed in what precedes, up to the best of our knowledge, the question of controllability to non-trivial trajectories (or even non-trivial stationary states) for parabolic free boundary problems such as (1.1), (1.4), (1.5) has not been addressed in the literature. We aim to present some of the difficulties which appear in solving this kind of control problem through this work.

1.2. Scope. In Section 2, we reformulate the control problem (1.1) on the timeindependent domain $(0,1)$. We give the linearization of $(1.1)$ around the target trajectory (see Section 5 for the general linearization and a brief discussion on the possible strategies for the general controllability to trajectories problem). In Section 3, we prove the null-controllability of the system linearized around $(\bar{v}, \bar{\ell})$. The PDE component is a linear heat equation with a source term, and the ODE component is simply an integrator of the heat solution's Dirichlet trace. The controllability requirement for the second component may thus be seen as a finite-dimensional constraint on the control. An improved observability inequality along with an adaptation of the HUM method provide the desired controllability result for both components of the linearized system. In Section 4, we come back to the nonlinear problem by means of a Banach fixed point argument.

\section{Reformulation of the PRoblem}

Transformation. To take advantage of a simplified functional setting, it is more advantageous to reformulate (1.1) in a domain which is time-independent. In view of linearizing, perturbations around the target trajectory would be defined in the same domain.

To this end, let us define the pull-back velocity function $w:(0,1) \rightarrow \mathbb{R}$ by

$$
w(t, x)=v(t, z), \quad x=\frac{z}{\ell(t)} \quad \text { for } x \in(0,1) .
$$

A simple application of the chain rule gives the following system of equations for $w$ :

$$
\begin{cases}w_{t}-\frac{1}{\ell^{2}} w_{x x}-\frac{\ell^{\prime}}{\ell} x w_{x}+\frac{1}{\ell} w w_{x}=0 & \text { in }(0, T) \times(0,1) \\ w(t, 0)=u(t), \quad w_{x}(t, 1)=0 & \text { in }(0, T) \\ \ell^{\prime}(t)=w(t, 1) & \text { in }(0, T) \\ w(0, x)=w_{0}(x), \quad \ell(0)=\ell_{0} & \text { in }(0,1),\end{cases}
$$

where $w_{0}(x)=v_{0}\left(\ell_{0} x\right)$. As $(1.1)$ and $(2.2)$ are equivalent provided $\ell(t)>0$ in $[0, T]$, we will henceforth concentrate our controllability analysis on the latter system.

Linearization. We shall now linearize the transformed system (2.2) around the target trajectory $(\bar{v}, \bar{\ell})$ given in (1.2). In order to illustrate some key difficulties related to the controllability to general trajectories for free boundary problems such as (1.1), we postpone to Section 5.1 the linearization of (2.2) around an arbitrary smooth timedependent trajectory $(\bar{w}, \bar{\ell})$, associated to initial and boundary data $\left(\bar{w}_{0}, \bar{\ell}_{0}, \bar{u}\right)$.

To proceed with the linearization around $(\bar{v}, \bar{\ell})$, we write $w=\bar{v}+y$ and $\ell=\bar{\ell}+h$, and keep all the terms which are linear with respect to $(y, h)$. The nonlinear problem 
satisfied by the perturbation variables reads

$$
\begin{cases}y_{t}-a y_{x x}+b y_{x}=\mathcal{N}(y, h) & \text { in }(0, T) \times(0,1) \\ y(t, 0)=u(t)-\bar{v}, \quad y_{x}(t, 1)=0 & \text { in }(0, T) \\ h^{\prime}(t)=y(t, 1) & \text { in }(0, T) \\ y(0, x)=y_{0}(x), \quad h(0)=h_{0} & \text { in }(0,1) .\end{cases}
$$

where $y_{0}(\cdot)=w_{0}(\cdot)-\bar{v}, h_{0}=\ell_{0}-\bar{\ell}(0)$, and the smooth, bounded coefficients are given by

$$
a(t)=\frac{1}{\bar{\ell}(t)^{2}}, \quad b(t, x)=\frac{\bar{v}-\bar{\ell}^{\prime}(t) x}{\bar{\ell}(t)} \quad \text { in } \quad[0, T] \times[0,1] .
$$

and the nonlinear term is of the form

$$
\mathcal{N}(y, h)=a\left(-h^{2} y_{t}-2 h \bar{\ell} y_{t}+h^{\prime} h x y_{x}+h^{\prime} \bar{\ell} x y_{x}+\bar{\ell} h x y_{x}-h y y_{x}-h \bar{v} y_{x}-\bar{\ell} y y_{x}\right) .
$$

It is important to note that since $\bar{\ell}^{\prime}(t)=\bar{v}$, from $(2.4)$ it follows that $b(t, 1)=0$. Moreover, the nonlinearity $\mathcal{N}(\cdot, \cdot)$ only consists of (at least) quadratic terms, which will facilitate the application of a Banach fixed point argument. The linearized problem corresponds to $(2.3)$ with $\mathcal{N} \equiv 0$.

Remark 2.1. At this point we notice that the linearized problem, namely (2.3) with $\mathcal{N} \equiv 0$, the PDE component $y$ and the ODE component $h$ are decoupled - namely, $y$ can be solved independently of $h$, and thus the coupling between the PDE and ODE is done solely through the nonlinear term. As seen in Section 5.1, the problem linearized around an arbitrary trajectory, namely (5.1), contains the terms $d h^{\prime}$ and eh, which are non-local as they may be expressed in terms of the Dirichlet trace of $y$ at $x=1$. As these terms act on a single point in space, at the level of the adjoint problem one could expect to obtain a non-local integral boundary condition over all points in space (see (5.3)). See Section 5.1 for more details.

Distributed control problem. Taking the previous transformations into account, Theorem 1.1 would in essence be a consequence of the null-controllability of System (2.3). To prove the latter, using common methodology for parabolic equations, we will first consider the distributed control problem

$$
\begin{cases}y_{t}-a y_{x x}+b y_{x}=\mathcal{N}(y, h)+u \mathbf{1}_{\omega} & \text { in }(0, T) \times(-1,1) \\ y(t,-1)=y_{x}(t, 1)=0 & \text { in }(0, T) \\ h^{\prime}(t)=y(t, 1) & \text { in }(0, T) \\ y(0, x)=y_{0}(x), \quad h(0)=h_{0} & \text { in }(-1,1)\end{cases}
$$

where $\omega \subsetneq(-1,0)$ is an open and non-empty interval. The initial datum $y_{0} \in H^{1}(0,1)$ is also extended to a datum $\widetilde{y}_{0}$ with $\left\|\widetilde{y}_{0}\right\|_{H^{1}(-1,1)} \leqslant\left\|y_{0}\right\|_{H^{1}(0,1)}$. By abuse of notation, we continue denoting the extended initial datum by $y_{0}$. Once the null-controllability problem for $(2.5)$ is solved, $u(t):=y(t, 0)+\bar{v}$ would provide the desired control for Problem (2.2), which in view of the previous discussion, also provides a solution to (1.1). 
To prove the null-controllability for system (2.5), we will first consider the associated linear system

$$
\begin{cases}y_{t}-a y_{x x}+b y_{x}=f+u \mathbf{1}_{\omega} & \text { in }(0, T) \times(-1,1) \\ y(t,-1)=y_{x}(t, 1)=0 & \text { in }(0, T) \\ h^{\prime}(t)=y(t, 1) & \text { in }(0, T) \\ y(0, x)=y_{0}(x), \quad h(0)=h_{0} & \text { in }(-1,1),\end{cases}
$$

where $f$ is a given source term. The null-controllability at time $T$ of the linearized system is the goal of the next section. The nonlinear term appearing in (2.5) will be seen as a small perturbation and will be dealt with by means of a Banach fixed-point argument.

\section{Null-Controllability of the linearized System}

In this Section, given $T>0$, arbitrarily large initial data $\left(y_{0}, \ell_{0}\right)$, and a source term $f$ with appropriate decay as $t \nearrow T$, we seek a trajectory $(y, h)$ of the linearized problem (2.6) satisfying

$$
y(T, \cdot)=0 \quad \text { in }(-1,1) \quad \text { and } \quad h(T)=0 .
$$

In (2.6) we are dealing with a cascade-like system, as knowing $y$ immediately yields $h$, with the latter being reduced to the integrator

$$
h(t)=h_{0}+\int_{0}^{t} y(\tau, 1) \mathrm{d} \tau .
$$

In other words, the null-controllability of $(2.6)$, would follow from solving the linear control problem (recall that $a(t)>0$ and $b(t, 1)=0$ )

$$
\begin{cases}y_{t}-a y_{x x}+b y_{x}=f+u \mathbf{1}_{\omega} & \text { in }(0, T) \times(-1,1) \\ y(t,-1)=y_{x}(t, 1)=0 & \text { in }(0, T) \\ y(0, x)=y_{0}(x) & \text { in }(-1,1) \\ y(T, x)=0 & \text { in }(-1,1)\end{cases}
$$

subject to the linear finite-dimensional constraint

$$
h_{0}+\int_{0}^{T} y(\tau, 1) \mathrm{d} \tau=0 .
$$

We will see this as a constrained controllability problem, namely with a linear finitedimensional constraint on the control $u$.

Carleman weights. Let us recall that $\omega=\left(\gamma_{1}, \gamma_{2}\right) \subsetneq(-1,0)$. We take $\left(a_{0}, b_{0}\right)$ with $\gamma_{1}<a_{0}<b_{0}<\gamma_{2}$ and introduce a function $\alpha_{0} \in C^{2}([-1,1])$ such that

$$
\alpha_{0}(x)>0 \quad \text { in }(-1,1), \quad \alpha_{0}( \pm 1)=0, \quad\left|\alpha_{0, x}\right|>0 \quad \text { in }(-1,1) \backslash\left(a_{0}, b_{0}\right),
$$

and for $\lambda \geqslant 1$ consider the function $\alpha$ defined by

$$
\alpha(t, x)=\theta(t)\left(e^{2 \lambda\left\|\alpha_{0}\right\|_{L} \infty}-e^{\lambda \alpha_{0}(x)}\right), \quad \text { in }(0, T) \times(-1,1),
$$


where $\theta \in C^{2}([0, T))$ is given by

$$
\theta(t)= \begin{cases}\frac{4}{T^{2}} & \text { on }\left[0, \frac{T}{2}\right] \\ \frac{1}{t(T-t)} & \text { on }\left[\frac{T}{2}, T\right) .\end{cases}
$$

Notice that the weight $\theta(t)$ does not blow up as $t \searrow 0$. This is because in view of the fixed-point argument, we will need to work with source-terms which do not vanish at $t=0$.

The main goal of this section is to prove the following result.

Theorem 3.1. Let $T>0$ be given. There exists $s \geqslant 1$ such that for any data $y_{0} \in$ $L^{2}(-1,1), h_{0} \in \mathbb{R}$ and $f \in L^{2}\left(0, T ; L^{2}(-1,1)\right)$ with

$$
\int_{0}^{T} \int_{-1}^{1} \theta^{-3} e^{2 s \alpha}|f|^{2} \mathrm{~d} x \mathrm{~d} t<\infty
$$

there exists a control $u \in L^{2}\left(0, T ; L^{2}(\omega)\right)$ such that the associated solution

$$
y \in L^{2}\left(0, T ; H^{1}(-1,1)\right) \cap C^{0}\left([0, T] ; L^{2}(-1,1)\right) \quad \text { and } \quad h \in H^{1}(0, T)
$$

of Problem (2.6) satisfies $y(T, \cdot)=0$ and $h(T)=0$. Moreover,

$$
\begin{aligned}
\|u\|_{L^{2}\left(0, T ; L^{2}(\omega)\right)} & +\left\|e^{s \alpha} y\right\|_{L^{2}\left(0, T ; L^{2}(-1,1)\right)} \\
& \leqslant C\left(\left\|y_{0}\right\|_{L^{2}(-1,1)}+\left|h_{0}\right|+\left\|\theta^{-3 / 2} e^{s \alpha} f\right\|_{L^{2}\left(0, T ; L^{2}(-1,1)\right)}\right)
\end{aligned}
$$

holds for some $C=C(T, \omega, s)>0$.

It is well known that a Carleman inequality (see Lemma 3.1) along with the HUM method yield the null-controllability of the linear heat equation (3.1) with a source term $f$ as in (3.4).

To control the second component $h$ to zero at time $T$, we will reformulate the constraint (3.2) by introducing an augmented adjoint problem for the heat equation with a nonhomogeneous boundary condition at $x=1$. The requirement $h(T)=0$ may then be achieved by adding a corrector term to the HUM control for the heat equation. To guarantee the existence of this control by means of the HUM method, we will need to prove an improved observability inequality. This idea appears in the work of Nakoulima [30] (see also [11] for a recent generalization), and has been applied in works on fluid-structure interaction problems (see [4, 14] for instance) where the structure's displacement at time $T$ is deduced after having controlled the fluid and structure velocities.

3.1. An improved observability inequality. We will make use of the following Carleman inequality for solutions to (recall that $b(t, 1)=0$ ) the adjoint heat equation

$$
\begin{cases}-\zeta_{t}-a \zeta_{x x}-(b \zeta)_{x}=g & \text { in }(0, T) \times(-1,1) \\ \zeta(t,-1)=\zeta_{x}(t, 1)=0 & \text { in }(0, T) \\ \zeta(T, x)=\zeta_{T}(x) & \text { in }(-1,1),\end{cases}
$$

and the weights defined in (3.3). The proof follows by combining the well-known Carleman inequality shown in Fursikov \& Imanuvilov [18, Lemma 1] (see also [35]) 
with the parameters $s \geqslant s_{0} \geqslant 1$ and $\lambda \geqslant \lambda_{0} \geqslant 1$ appearing therein being henceforth fixed, and energy estimates (recall that $b(t, 1)=0$ ) as done in [15, Section 3].

Lemma 3.1. Let $T>0$. There exists $C=C(T, \omega, s, \lambda)>0$ such that for every datum $\zeta_{T} \in L^{2}(-1,1)$ and source $g \in L^{2}\left(0, T ; L^{2}(-1,1)\right)$, the unique weak solution $\zeta \in L^{2}\left(0, T ; H^{1}(-1,1)\right) \cap C^{0}\left([0, T] ; L^{2}(-1,1)\right)$ to (3.6) satisfies

$$
\begin{aligned}
& \int_{0}^{T} \int_{-1}^{1} \theta^{3} e^{-2 s \alpha}|\zeta|^{2} \mathrm{~d} x \mathrm{~d} t+\int_{-1}^{1}|\zeta(0, x)|^{2} \mathrm{~d} x \\
& \leqslant C\left(\int_{0}^{T} \int_{-1}^{1} e^{-2 s \alpha}|g|^{2} \mathrm{~d} x \mathrm{~d} t+\int_{0}^{T} \int_{\omega} \theta^{3} e^{-2 s \alpha}|\zeta|^{2} \mathrm{~d} x \mathrm{~d} t\right) .
\end{aligned}
$$

The Carleman inequality (3.7) guarantees the coercivity and continuity of the strictly convex HUM functional, the unique minimizer of which yields a solution to the adjoint heat equation (3.6) and subsequently a solution to the control problem (3.1) after investigating the corresponding Euler-Lagrange equation.

To take care of the constraint $h(T)=0$, let us consider the augmented adjoint problem

$$
\begin{cases}-\psi_{t}-a \psi_{x x}-(b \psi)_{x}=0 & \text { in }(0, T) \times(-1,1) \\ \psi(t,-1)=0, \quad \psi_{x}(t, 1)=1 & \text { in }(0, T) \\ \psi(T, x)=0 & \text { in }(-1,1) .\end{cases}
$$

Multiplying the heat equation appearing in System (2.6) by the unique weak solution $\psi \in L^{2}\left(0, T ; H^{1}(-1,1)\right) \cap C^{0}\left([0, T] ; L^{2}(-1,1)\right)$ of $(3.8)$ and integrating, we see that due to (3.2), a control $u$ is such that the corresponding solution of $(2.6)$ satisfies $h(T)=0$ if and only if

$$
\int_{0}^{T} \int_{\omega} u \psi \mathrm{d} x \mathrm{~d} t=-\int_{-1}^{1} y_{0}(x) \psi(0, x) \mathrm{d} x+h_{0}-\int_{0}^{T} \int_{-1}^{1} f \psi \mathrm{d} x \mathrm{~d} t .
$$

Let us define the projector

$$
\mathbb{P}(\zeta):=\frac{\int_{(0, T) \times \omega} \psi \zeta \mathrm{d} x \mathrm{~d} t}{\int_{(0, T) \times \omega}|\psi|^{2} \mathrm{~d} x \mathrm{~d} t} \quad \text { for all } \zeta \in L^{2}\left(0, T ; L^{2}(-1,1)\right) .
$$

The key property of the operator $\mathbb{P}(\cdot)$ is its finite-dimensional range (in fact, onedimensional range). Our next result is the desired improved observability inequality. The proof follows an indirect, compactness-uniqueness argument (following ideas in $[4,14])$. We assume the setting of Lemma 3.1.

Proposition 3.1. There exists a constant $C_{\mathrm{obs}}=C_{\mathrm{obs}}(T, \omega, s, \lambda)>0$ such that for every datum $\zeta_{T} \in L^{2}(-1,1)$ and source $g \in L^{2}\left(0, T ; L^{2}(-1,1)\right)$, the unique weak solution $\zeta \in L^{2}\left(0, T ; H^{1}(-1,1)\right) \cap C^{0}\left([0, T] ; L^{2}(-1,1)\right)$ to (3.6) satisfies

$$
\begin{aligned}
\int_{0}^{T} \int_{-1}^{1} \theta^{3} e^{-2 s \alpha}|\zeta|^{2} \mathrm{~d} x \mathrm{~d} t+\int_{-1}^{1}|\zeta(0, x)|^{2} \mathrm{~d} x+|\mathbb{P}(\zeta)|^{2} \\
\leqslant C_{\mathrm{obs}}\left(\int_{0}^{T} \int_{-1}^{1} e^{-2 s \alpha}|g|^{2} \mathrm{~d} x \mathrm{~d} t+\int_{0}^{T} \int_{\omega}|\zeta-\mathbb{P}(\zeta) \psi|^{2} \mathrm{~d} x \mathrm{~d} t\right) .
\end{aligned}
$$


Proof. We will begin by showing by means of an indirect argument that

$$
\begin{aligned}
& \int_{0}^{T} \int_{-1}^{1} \theta^{3} e^{-2 s \alpha}|\zeta|^{2} \mathrm{~d} x \mathrm{~d} t+\int_{-1}^{1}|\zeta(0, x)|^{2} \mathrm{~d} x \\
& \leqslant C_{2}\left(\int_{0}^{T} \int_{-1}^{1} e^{-2 s \alpha}|g|^{2} \mathrm{~d} x \mathrm{~d} t+\int_{0}^{T} \int_{\omega}|\zeta-\mathbb{P}(\zeta) \psi|^{2} \mathrm{~d} x \mathrm{~d} t\right)
\end{aligned}
$$

for some $C_{2}=C_{2}(T, \omega, s, \lambda)>0$ and any $\left(\zeta_{T}, g\right)$ as in the statement, which would cover the two leftmost terms of the desired inequality (3.10). To do so, let us assume by contradiction that (3.11) is false, thus there exist two sequences $\left\{\zeta_{T}^{k}\right\}_{k=1}^{\infty}$ and $\left\{g^{k}\right\}_{k=1}^{\infty}$ such that

$$
\begin{aligned}
1 & =\int_{0}^{T} \int_{-1}^{1} \theta^{3} e^{-2 s \alpha}\left|\zeta^{k}\right|^{2} \mathrm{~d} x \mathrm{~d} t+\int_{-1}^{1}\left|\zeta^{k}(0, \cdot)\right|^{2} \mathrm{~d} x \\
& \geqslant k\left(\int_{0}^{T} \int_{-1}^{1} e^{-2 s \alpha}\left|g^{k}\right|^{2} \mathrm{~d} x \mathrm{~d} t+\int_{0}^{T} \int_{\omega}\left|\zeta^{k}-\mathbb{P}\left(\zeta^{k}\right) \psi\right|^{2} \mathrm{~d} x \mathrm{~d} t\right),
\end{aligned}
$$

for any $k \in \mathbb{N}$, with $\zeta^{k}$ being the corresponding solution to the adjoint problem (3.6). Elementary inequalities give

$$
\begin{aligned}
& \frac{1}{2} \int_{0}^{T} \int_{\omega} \theta^{3} e^{-2 s \alpha}\left|\mathbb{P}\left(\zeta^{k}\right) \psi\right|^{2} \mathrm{~d} x \mathrm{~d} t \\
& \quad \leqslant \int_{0}^{T} \int_{\omega} \theta^{3} e^{-2 s \alpha}\left|\zeta^{k}\right|^{2} \mathrm{~d} x \mathrm{~d} t+\int_{0}^{T} \int_{\omega} \theta^{3} e^{-2 s \alpha}\left|\zeta^{k}-\mathbb{P}\left(\zeta^{k}\right) \psi\right|^{2} \mathrm{~d} x \mathrm{~d} t,
\end{aligned}
$$

thus the left-most integral is uniformly bounded for any $k \in \mathbb{N}$ in view of (3.12) (recall also the definition of the weights in (3.3)). Hence, $\mathbb{P}\left(\zeta^{k}\right)$ is uniformly bounded in $\mathbb{R}$ with respect to $k \in \mathbb{N}$, whence it follows that

$$
\mathbb{P}\left(\zeta^{k}\right) \longrightarrow \mathbb{P}_{*} \quad \text { as } k \longrightarrow \infty
$$

for some $\mathbb{P}_{*} \in \mathbb{R}$, along some subsequence. From (3.12), we see that the functions $\zeta^{k}$ and $\zeta^{k}(0, \cdot)$ are uniformly bounded in $L^{2}\left(0, T-\varepsilon ; L^{2}(-1,1)\right)$ and $L^{2}(-1,1)$ respectively, for all $\varepsilon>0$, as well as

$$
\int_{0}^{T-\varepsilon} \int_{-1}^{1}\left|g^{k}\right|^{2} \mathrm{~d} x \mathrm{~d} t \lesssim \frac{1}{k}
$$

Whence, using the well-known energy estimates for the heat equation (recall that $b(t, 1)=0)$, one also has that

$$
\begin{array}{ll}
\zeta^{k} \rightarrow \zeta & \text { weakly in } L^{2}\left(0, T-\varepsilon ; H^{1}(-1,1)\right) \\
\zeta_{t}^{k} \rightarrow \zeta_{t} & \text { weakly in } L^{2}\left(0, T-\varepsilon ; H^{-1}(-1,1)\right)
\end{array}
$$

along subsequences as $k \longrightarrow \infty$. It can thus be seen that $\zeta$ satisfies

$$
\begin{cases}-\zeta_{t}-a \zeta_{x x}-(b \zeta)_{x}=0 & \text { in }(0, T) \times(-1,1) \\ \zeta(t,-1)=0, \quad \zeta_{x}(t, 1)=0 & \text { in }(0, T) .\end{cases}
$$

In $(0, T) \times \omega$, we have $\zeta^{k}=\left(\zeta^{k}-\mathbb{P}\left(\zeta^{k}\right) \psi\right)+\mathbb{P}\left(\zeta^{k}\right) \psi$, so in view of (3.12) and (3.13) we have

$$
\zeta^{k} \longrightarrow \mathbb{P}_{*} \psi \quad \text { strongly in } L^{2}\left(0, T ; L^{2}(\omega)\right)
$$

as $k \longrightarrow \infty$. The above convergence implies that $\zeta=\mathbb{P}_{*} \psi$ in $(0, T) \times \omega$. As $\psi$ is also in the kernel of the heat operator (thus, so is $\mathbb{P}_{*} \psi$ ), by unique continuation we deduce 
that $\zeta=\mathbb{P}_{*} \psi$ in $(0, T) \times(-1,1)$. But this can only hold if $\zeta \equiv 0$ and $\mathbb{P}_{*}=0$, since $\psi_{x}(t, 1)=1$.

From (3.14), we may thus deduce that

$$
\zeta^{k} \longrightarrow 0 \quad \text { strongly in } L^{2}\left(0, T ; L^{2}(\omega)\right)
$$

as $k \longrightarrow \infty$, and using (3.7) (noting that (3.12) is used for $g^{k}$ ) we deduce that

$$
\int_{0}^{T} \int_{-1}^{1} \theta^{3} e^{-2 s \alpha}\left|\zeta^{k}\right|^{2} \mathrm{~d} x \mathrm{~d} t+\int_{-1}^{1}\left|\zeta^{k}(0, x)\right|^{2} \mathrm{~d} x \longrightarrow 0
$$

as $k \longrightarrow \infty$, which contradicts (3.12). Consequently, (3.11) holds. Arguing as for (3.11), we can show

$$
\left|\int_{0}^{T} \int_{\omega} \theta^{3} e^{-2 s \alpha} \zeta \psi \mathrm{d} x \mathrm{~d} t\right|^{2} \leqslant C_{5}\left(\int_{0}^{T} \int_{-1}^{1} e^{-2 s \alpha}|g|^{2} \mathrm{~d} x \mathrm{~d} t+\int_{0}^{T} \int_{\omega}|\zeta-\mathbb{P}(\zeta) \psi|^{2} \mathrm{~d} x \mathrm{~d} t\right)
$$

for some $C_{5}=C_{5}(T, \omega, s)>0$. Indeed, setting up an assumption for (3.15) as in (3.12) and applying Cauchy-Schwarz, after following the lines of the previous step, it may be seen that this would provide the necessary contradiction.

Remark 3.1. While Proposition 3.1 yields the desired improved observability inequality for what follows, due to the indirect argument used for the proof an explicit dependence of the newly obtained constant on the parameters $(T, \omega)$ is not guaranteed.

3.2. Proof of Theorem 3.1. We are now in a position to complete the proof of Theorem 3.1, which follows by adapting the well-known HUM arguments.

Proof of Theorem 3.1. For a solution $\psi$ of (3.8), let us henceforth denote

$$
M_{0}:=-\int_{-1}^{1} y_{0}(x) \psi(0, \cdot) \mathrm{d} x+h_{0}-\int_{0}^{T} \int_{-1}^{1} f \psi \mathrm{d} x \mathrm{~d} t .
$$

We split the proof in three steps.

Step 1: Minimization problem. Consider the functional

$$
\begin{aligned}
J_{\text {obs }}\left(\zeta_{T}, g\right):= & \frac{1}{2} \int_{0}^{T} \int_{\omega}|\zeta-\mathbb{P}(\zeta) \psi|^{2} \mathrm{~d} x \mathrm{~d} t+\frac{1}{2} \int_{0}^{T} \int_{-1}^{1} e^{-2 s \alpha}|g|^{2} \mathrm{~d} x \mathrm{~d} t \\
& -\int_{0}^{T} \int_{-1}^{1} f \zeta \mathrm{d} x \mathrm{~d} t-\int_{-1}^{1} y_{0}(x) \zeta(0, x) \mathrm{d} x-\mathbb{P}(\zeta) M_{0},
\end{aligned}
$$

initially defined for $\left(\zeta_{T}, g\right) \in L^{2}(-1,1) \times L^{2}\left(0, T ; L^{2}(-1,1)\right)$ with corresponding solution $\zeta \in L^{2}\left(0, T ; H^{1}(-1,1)\right) \cap C^{0}\left([0, T] ; L^{2}(-1,1)\right)$ to the adjoint heat equation (3.6), and $\psi$ being the solution to the augmented adjoint problem (3.8). We will show the existence of a minimizer to $J_{\text {obs }}$, which will consequently be used to build the desired control - state pair for Problem (2.6).

We remark that the quantity

$$
\left\|\left(\zeta_{T}, g\right)\right\|_{\text {obs }}^{2}=\int_{0}^{T} \int_{\omega}|\zeta-\mathbb{P}(\zeta) \psi|^{2} \mathrm{~d} x \mathrm{~d} t+\int_{0}^{T} \int_{-1}^{1} e^{-2 s \alpha}|g|^{2} \mathrm{~d} x \mathrm{~d} t
$$

defines a norm on $L^{2}(-1,1) \times L^{2}\left(0, T ; L^{2}(-1,1)\right)$. In order to have completeness, we thus introduce the space

$$
X_{\mathrm{obs}}:=\overline{L^{2}(-1,1) \times L^{2}\left(0, T ; L^{2}(-1,1)\right)}\|\cdot\|_{\mathrm{obs}} .
$$


The set $X_{\text {obs }}$ is then endowed with the Hilbert structure given by the above norm.

On $X_{\text {obs }}$, the functional $J_{\text {obs }}$ may be extended by continuity in a unique way. Indeed, the improved weighted observability inequality (3.10) implies (recall that $f$ is assumed to satisfy (3.4))

$$
\begin{aligned}
\left|\int_{0}^{T} \int_{-1}^{1} f \zeta \mathrm{d} x \mathrm{~d} t\right| & \leqslant\left(\int_{0}^{T} \int_{-1}^{1} \theta^{-3} e^{2 s \alpha}|f|^{2} \mathrm{~d} x \mathrm{~d} t\right)^{1 / 2}\left(\int_{0}^{T} \int_{-1}^{1} \theta^{3} e^{-2 s \alpha}|\zeta|^{2} \mathrm{~d} x \mathrm{~d} t\right)^{1 / 2} \\
& \leqslant C_{T}\left\|\theta^{-3 / 2} e^{s \alpha} f\right\|_{L^{2}\left(0, T ; L^{2}(-1,1)\right)}\left\|\left(\zeta_{T}, g\right)\right\|_{\text {obs }}
\end{aligned}
$$

as well as

$$
\begin{aligned}
\left|\int_{-1}^{1} y_{0}(x) \zeta(0, x) \mathrm{d} x\right| & \leqslant\left(\int_{-1}^{1}\left|y_{0}\right|^{2} \mathrm{~d} x\right)^{1 / 2}\left(\int_{-1}^{1}|\zeta(0, x)|^{2} \mathrm{~d} x\right)^{1 / 2} \\
& \leqslant C_{T}\left\|y_{0}\right\|_{L^{2}(-1,1)}\left\|\left(\zeta_{T}, g\right)\right\|_{\text {obs }}
\end{aligned}
$$

and

$$
|\mathbb{P}(\zeta)| \leqslant C_{T}\left\|\left(\zeta_{T}, g\right)\right\|_{\text {obs }}
$$

for some $C_{T}=C_{T}(\omega, s, \lambda)>0$. Due to (3.17) - (3.18) - (3.19), it can be seen that the functional $J_{\mathrm{obs}}$ is also coercive. As $J_{\mathrm{obs}}$ is also strictly convex on $X_{\mathrm{obs}}$ (since $\|\cdot\|_{\mathrm{obs}}$ is a Hilbert norm), it admits a unique minimizer $(\widehat{\zeta}, \widehat{g}) \in X_{\text {obs }}$ by the direct method in the calculus of variations.

Step 2: Null-controllability requirements. Now the unique minimizer $\left(\widehat{\zeta_{T}}, \widehat{g}\right) \in X_{\text {obs }}$ of $J_{\text {obs }}$ satisfies the Euler-Lagrange equation

$$
\begin{aligned}
0= & \int_{0}^{T} \int_{\omega}(\widehat{\zeta}-\mathbb{P}(\widehat{\zeta}) \psi) \varphi \mathrm{d} x \mathrm{~d} t+\int_{0}^{T} \int_{-1}^{1} e^{-2 s \alpha} \widehat{g} F \mathrm{~d} x \mathrm{~d} t \\
& -\int_{0}^{T} \int_{-1}^{1} f \varphi \mathrm{d} x \mathrm{~d} t-\int_{-1}^{1} y_{0}(x) \varphi(0, x) \mathrm{d} x-\mathbb{P}(\varphi) M_{0}
\end{aligned}
$$

for all $\left(\varphi_{T}, F\right) \in X_{\mathrm{obs}}$, where $\widehat{\zeta}$ and $\varphi$ denote the solutions to (3.6) corresponding to $\left(\widehat{\zeta_{T}}, \widehat{g}\right)$ and $\left(\varphi_{T}, F\right)$ respectively. Comparing (3.20) with (3.22), we are led to consider the control function

$$
u:=-(\widehat{\zeta}-\mathbb{P}(\widehat{\zeta}) \psi)+M_{0}\left(\int_{0}^{T} \int_{\omega} \psi^{2} \mathrm{~d} x \mathrm{~d} t\right)^{-1} \psi
$$

restricted to $\omega$, where $\psi$ is the unique solution to the augmented adjoint problem (3.8). Let $y \in L^{2}\left(0, T ; H^{1}(-1,1)\right) \cap C^{0}\left([0, T] ; L^{2}(-1,1)\right)$ be the solution to the heat equation in $(2.6)$ with control $u$. Let us justify this choice. Noting that

$$
\int_{0}^{T} \int_{\omega} u \varphi \mathrm{d} x \mathrm{~d} t=-\int_{0}^{T} \int_{\omega}(\widehat{\zeta}-\mathbb{P}(\widehat{\zeta}) \psi) \varphi \mathrm{d} x \mathrm{~d} t+\mathbb{P}(\varphi) M_{0}
$$

we come back to (3.20) and deduce that

$$
\begin{aligned}
0= & -\int_{0}^{T} \int_{-1}^{1} e^{2 s \alpha} \widehat{g} F \mathrm{~d} x \mathrm{~d} t+\int_{0}^{T} \int_{\omega} u \varphi \mathrm{d} x \mathrm{~d} t \\
& +\int_{0}^{T} \int_{-1}^{1} f \varphi \mathrm{d} x \mathrm{~d} t+\int_{-1}^{1} y_{0} \varphi(0, \cdot) \mathrm{d} x .
\end{aligned}
$$


On the other hand, multiplying the heat component in (2.6) by any $\varphi$ weak solution of (3.6) with initial data $\varphi_{T}$ and source term $F$, we see (modulo a density argument) that

$$
\int_{-1}^{1} y(T, \cdot) \varphi_{T} \mathrm{~d} x=-\int_{0}^{T} \int_{-1}^{1}(y F+f \varphi) \mathrm{d} x \mathrm{~d} t+\int_{-1}^{1} y_{0} \varphi(0, \cdot) \mathrm{d} x+\int_{0}^{T} \int_{\omega} u \varphi \mathrm{d} x \mathrm{~d} t .
$$

Comparing with $(3.21)$, for all $\left(\varphi_{T}, F\right) \in L^{2}(-1,1) \times L^{2}\left(0, T ; L^{2}(-1,1)\right)$ one has

$$
\int_{-1}^{1} y(T, \cdot) \varphi_{T} \mathrm{~d} x=\int_{0}^{T} \int_{-1}^{1}\left(e^{2 s \alpha} \widehat{g}-y\right) F \mathrm{~d} x \mathrm{~d} t .
$$

As $F$ is arbitrary, choosing $F \equiv 0$, we get the desired control requirement $y(T, \cdot)=0$. On the other hand, as $\varphi_{T}$ is arbitrary, choosing $\varphi_{T} \equiv 0$, we see also that

$$
y=\widehat{g} e^{-2 s \alpha} \text {. }
$$

We now define $h \in H^{1}(0, T)$ by

$$
h(t):=h_{0}+\int_{0}^{t} y(\tau, 1) \mathrm{d} \tau .
$$

It remains to be seen that the above-defined control $u$ is such that $h(T)=0$. Recalling the definition of $M_{0}$ in (3.16), a straightforward computation shows that

$$
\int_{0}^{T} \int_{\omega} u \psi \mathrm{d} x \mathrm{~d} t=M_{0}
$$

which in view of (3.9) yields the conclusion $h(T)=0$, as desired.

Step 3: Estimates. As $J_{\mathrm{obs}}\left(\widehat{\zeta}_{T}, \widehat{g}\right) \leqslant J_{\mathrm{obs}}(0,0)=0$, straightforward estimates along with $(3.17)$ - (3.19) give

$$
\begin{aligned}
\| \widehat{\zeta}-\mathbb{P}(\widehat{\zeta}) \psi & \left\|_{L^{2}\left(0, T ; L^{2}(\omega)\right)}+\right\| e^{-s \alpha} \widehat{g} \|_{L^{2}\left(0, T ; L^{2}(-1,1)\right)} \\
& \leqslant C_{1}\left(\left\|y_{0}\right\|_{L^{2}(-1,1)}+\left|h_{0}\right|+\left\|\theta^{-3 / 2} e^{s \alpha} f\right\|_{L^{2}\left(0, T ; L^{2}(-1,1)\right)}\right)
\end{aligned}
$$

for some $C_{1}=C_{1}(T, \omega, s, \lambda)>0$. On another hand, it may easily be checked that

$$
\int_{0}^{T} \int_{\omega} u^{2} \mathrm{~d} x \mathrm{~d} t=\int_{0}^{T} \int_{\omega}(\widehat{\zeta}-\mathbb{P}(\widehat{\zeta}) \psi)^{2} \mathrm{~d} x \mathrm{~d} t+M_{0}^{2}\left(\int_{0}^{T} \int_{\omega} \psi^{2} \mathrm{~d} x \mathrm{~d} t\right)^{-1}
$$

Thus, in view of the definitions of the control $u$ and the state $y$ and (3.23) and (3.24) lead us to conclude that

$$
\begin{aligned}
\|u\|_{L^{2}\left(0, T ; L^{2}(\omega)\right)} & +\left\|e^{s \alpha} y\right\|_{L^{2}\left(0, T ; L^{2}(-1,1)\right)} \\
& \leqslant C_{2}\left(\left\|y_{0}\right\|_{L^{2}(-1,1)}+\left|h_{0}\right|+\left\|\theta^{-3 / 2} e^{s \alpha} f\right\|_{L^{2}\left(0, T ; L^{2}(-1,1)\right)}\right)
\end{aligned}
$$

for some $C_{2}=C_{2}(T)>0$. This concludes the proof.

The following Lemma gives additional estimates of the controlled trajectory in the weighted spaces provided more regular initial data. 
Lemma 3.2. Let $(v, y, h)$ denote the control-state pair given by Theorem 3.1. Assume moreover that $y_{0} \in H^{1}(-1,1)$. Then

$$
\begin{aligned}
\left\|\theta^{-1} e^{s \alpha} y_{x}\right\|_{L^{2}\left(0, T ; L^{2}(-1,1)\right)} & +\left\|\theta^{-2} e^{s \alpha} y_{t}\right\|_{L^{2}\left(0, T ; L^{2}(-1,1)\right)} \\
& +\left\|\theta^{-2} e^{s \alpha} y_{x x}\right\|_{L^{2}\left(0, T ; L^{2}(-1,1)\right)}+\left\|\theta^{-2} e^{s \alpha} y\right\|_{L^{\infty}\left(0, T ; H^{1}(-1,1)\right)} \\
& \leqslant C\left(\left\|y_{0}\right\|_{H^{1}(-1,1)}+\left|h_{0}\right|+\left\|\theta^{-3 / 2} e^{s \alpha} f\right\|_{L^{2}\left(0, T ; L^{2}(-1,1)\right)}\right)
\end{aligned}
$$

holds for some $C=C(T, \omega, s)>0$.

Proof. The proof for estimating the first three norms follows standard energy estimate arguments, and we refer to [16, Lemma 3.4] for details. To obtain the weighted $L^{\infty}\left(H^{1}\right)$-estimate, we note that by interpolation

$$
\left\|\theta^{-2} e^{s \alpha} y\right\|_{L^{\infty}\left(0, T ; H^{1}(-1,1)\right)} \lesssim\left\|\theta^{-2} e^{s \alpha} y\right\|_{L^{2}\left(0, T ; H^{2}(-1,1)\right)}^{1 / 2}\left\|\theta^{-2} e^{s \alpha} y\right\|_{H^{1}\left(0, T ; L^{2}(-1,1)\right)}^{1 / 2},
$$

and the right-hand side is bounded by the properties of the Carleman weights and the three previous estimates.

\section{THE NONLINEAR PROBLEM}

We now look to conclude the proof of Theorem 1.1 by virtue of a fixed-point argument for nonlinear system

$$
\begin{cases}y_{t}-a y_{x x}+b y_{x}=\mathcal{N}(y, h)+u \mathbf{1}_{\omega} & \text { in }(0, T) \times(-1,1) \\ y(t,-1)=y_{x}(t, 1)=0 & \text { in }(0, T) \\ h^{\prime}(t)=y(t, 1) & \text { in }(0, T) \\ y(0, x)=y_{0}(x), \quad h(0)=h_{0} & \text { in }(-1,1),\end{cases}
$$

a restriction argument and reverting the transformations performed in Section 2. We recall that the nonlinear term in (4.1) is of the form

$$
\mathcal{N}(y, h)=a\left(-h y_{t}(h+2 \bar{\ell})+h^{\prime} y_{x}(h x+\bar{\ell} x)+h y_{x}(\bar{\ell} x+\bar{v})-y y_{x}(h+\bar{\ell})\right),
$$

only consisting of (at least) quadratic terms.

Let us consider the norm

$$
\begin{aligned}
\|y\|_{\mathcal{Y}}: & =\left\|e^{s \alpha} y\right\|_{L^{2}\left(0, T ; L^{2}(-1,1)\right)}+\left\|\theta^{-1} e^{s \alpha} y_{x}\right\|_{L^{2}\left(0, T ; L^{2}(-1,1)\right)} \\
& +\left\|\theta^{-2} e^{s \alpha} y_{t}\right\|_{L^{2}\left(0, T ; L^{2}(-1,1)\right)}+\left\|\theta^{-2} e^{s \alpha} y_{x x}\right\|_{L^{2}\left(0, T ; L^{2}(-1,1)\right)} \\
& +\left\|\theta^{-2} e^{s \alpha} y\right\|_{L^{\infty}\left(0, T ; H^{1}(-1,1)\right)} .
\end{aligned}
$$

We begin by the following lemma, which provides the appropriate estimates of each nonlinear term with respect to the $\|\cdot\| \mathcal{Y}$ - norm.

Lemma 4.1 (Nonlinear estimates). For $y_{0} \in H^{1}(-1,1)$, let $(y, h)$ denote the controlled trajectory of the linearized problem (2.6) given by Theorem 3.1. Then

$$
\left\|\theta^{-3 / 2} e^{s \alpha} \mathcal{N}(y, h)\right\|_{L^{2}\left(0, T ; L^{2}(-1,1)\right)} \leqslant C\|y\|_{\mathcal{Y}}^{2}
$$

holds for some $C=C(T, \omega, s)>0$. 
Proof. We begin by noting that $a \in L^{\infty}(0, T)$. Using interpolation estimates,

$$
\|y\|_{L^{\infty}\left(L^{\infty}\right)} \lesssim\|y\|_{L^{\infty}\left(H^{1}\right)} \lesssim\|y\|_{H^{1}\left(L^{2}\right)}^{1 / 2}\|y\|_{L^{2}\left(H^{2}\right)}^{1 / 2} \lesssim\|y\|_{\mathcal{Y}} .
$$

Let us begin by estimating the right-most term of (4.2). Since $h+\bar{\ell} \in L^{\infty}(0, T)$ as well as $\theta^{-1} \in L^{\infty}(0, T)$, using (4.3) one deduces

$$
\begin{aligned}
\left\|\theta^{-3 / 2} e^{s \alpha}(h+\bar{\ell}) y y_{x}\right\|_{L^{2}\left(0, T ; L^{2}(-1,1)\right)} & \lesssim\|y\|_{L^{\infty}\left(L^{\infty}\right)}\left\|\theta^{-3 / 2} e^{s \alpha} y_{x}\right\|_{L^{2}\left(0, T ; L^{2}(-1,1)\right)} \\
& \lesssim\|y\|_{\mathcal{Y} .}^{2}
\end{aligned}
$$

To estimate the two middle terms in (4.2), we first observe that since $h(T)=0$, for any $t \in[0, T]$ we may write

$$
h(t)=h(t)-h(T) \lesssim_{T} \sup _{t \in[0, T]}\left|h^{\prime}(t)\right| .
$$

Moreover, as $h^{\prime}(t)=y(t, 1)$ for $t \in(0, T),(h+\bar{\ell}) \cdot \in L^{\infty}((0, T) \times(-1,1))$ and $\bar{\ell} \cdot+\bar{v} \in$ $L^{\infty}((0, T) \times(-1,1))$ and $\theta^{-1} \in L^{\infty}(0, T)$, we may estimate the middle terms using (4.5) and (4.3) as follows:

$$
\begin{aligned}
\left\|\theta^{-3 / 2} e^{s \alpha} h^{\prime} y_{x}(h+\bar{\ell})\right\|_{L^{2}\left(0, T ; L^{2}(-1,1)\right)} & +\left\|\theta^{-3 / 2} e^{s \alpha} h y_{x}(\bar{\ell}+\bar{v})\right\|_{L^{2}\left(0, T ; L^{2}(-1,1)\right)} \\
& \lesssim\|y\|_{L^{\infty}\left(L^{\infty}\right)}\left\|e^{-3 / 2} e^{s \alpha} y_{x}\right\|_{L^{2}\left(0, T ; L^{2}(-1,1)\right)} \\
& \lesssim\|y\|_{\mathcal{Y}}^{2}
\end{aligned}
$$

To estimate the leftmost term, we need further arguments. Indeed, arguing as above we deduce

$$
\left\|\theta^{-3 / 2} e^{s \alpha} h y_{t}(h+2 \bar{\ell})\right\|_{L^{2}\left(0, T ; L^{2}(-1,1)\right)} \lesssim\left\|\theta^{1 / 2} h\right\|_{L^{\infty}(0, T)}\left\|\theta^{-2} e^{s \alpha} y_{t}\right\|_{L^{2}\left(0, T ; L^{2}(-1,1)\right)} .
$$

The desired estimate would thus follow provided

$$
\left\|\theta^{1 / 2} h\right\|_{L^{\infty}(0, T)} \lesssim\|y\|_{\mathcal{Y}}
$$

holds. To prove $(4.7)$, let $0<\bar{\alpha}<\min _{x \in(-1,1)}\left(e^{2 \lambda\left\|\alpha_{0}\right\|_{L^{\infty}}}-e^{\lambda \alpha_{0}}\right)$ and we first notice that since $h(T)=0$ and $e^{-\frac{s \bar{\alpha} \theta(T)}{2}}=0$, by the Cauchy mean-value theorem

$$
\left|\frac{h(t)}{e^{-\frac{s \bar{\alpha} \theta}{2}}}\right|=\left|\frac{h(t)-h(T)}{e^{-\frac{s \bar{\alpha} \theta(t)}{2}}-e^{-\frac{s \bar{\alpha} \theta(T)}{2}}}\right| \lesssim\left\|\frac{h^{\prime}}{\left(e^{-\frac{s \bar{\alpha} \theta}{2}}\right)^{\prime}}\right\|_{L^{\infty}(0, T)} \lesssim_{T}\left\|\frac{h^{\prime}}{e^{-\frac{s \bar{\alpha} \theta}{2}}}\right\|_{L^{\infty}(0, T)}
$$

for $t \in[0, T]$. We proceed in estimating the right-most term in (4.8). For $t \in[0, T]$, using trace estimates and the decay properties of the Carleman weights,

$$
\begin{aligned}
e^{s \bar{\alpha} \theta(t)}\left|h^{\prime}(t)\right|^{2} & =e^{s \bar{\alpha} \theta(t)}|y(t, 1)|^{2} \\
& \lesssim \sup _{t \in[0, T]} \int_{-1}^{1} e^{s \bar{\alpha} \theta(t)}|y(t, x)|^{2} \mathrm{~d} x+\sup _{t \in[0, T]} \int_{-1}^{1} e^{s \bar{\alpha} \theta(t)}\left|y_{x}(t, x)\right|^{2} \mathrm{~d} x \\
& \lesssim T \sup _{t \in[0, T]} \int_{-1}^{1} \theta^{-4} e^{2 s \alpha}|y|^{2} \mathrm{~d} x+\sup _{t \in[0, T]} \int_{-1}^{1} \theta^{-4} e^{2 s \alpha}\left|y_{x}\right|^{2} \mathrm{~d} x,
\end{aligned}
$$


and the right-most terms are bounded by Lemma 3.2. By (4.9), (4.8) holds, and the latter rewrites as

$$
|h(t)| \lesssim_{T} e^{-\frac{s \bar{\alpha} \theta(t)}{2}}\left\|\frac{h^{\prime}}{e^{-\frac{s \bar{\alpha} \theta}{2}}}\right\|_{L^{\infty}(0, T)} .
$$

Consequently, (4.10) along with the decay properties of the Carleman weights yield (4.7), which concludes the proof.

We are now in a position to state and prove the null-controllability result for Problem (4.1).

Theorem 4.1. Let $T>0$ and $\omega=\left(\gamma_{1}, \gamma_{2}\right) \subsetneq(-1,0)$ be non-empty. There exists $r>0$ such that for all $\left(y_{0}, h_{0}\right) \in H^{1}(-1,1) \times \mathbb{R}$ satisfying $\left\|y_{0}\right\|_{H^{1}(-1,1)}+\left|h_{0}\right| \leqslant r$, there exists a control $u \in L^{2}\left(0, T ; L^{2}(\omega)\right)$ such that the corresponding strong solution

$$
y \in L^{2}\left(0, T ; H^{2}(-1,1)\right) \cap C^{0}\left([0, T] ; H^{1}(-1,1)\right) \quad h \in H^{1}(0, T)
$$

of (4.1) satisfies $y(T, \cdot)=0$ in $(-1,1)$ and $h(T)=0$.

The proof follows a Banach fixed point argument. For $r>0$, we consider the associated ball of $H^{1}(-1,1)$ :

$$
\mathfrak{B}_{r}:=\left\{y_{0} \in H^{1}(-1,1):\left\|y_{0}\right\|_{H^{1}(-1,1)} \leqslant r\right\}
$$

and we also set

$$
\mathfrak{F}_{r}=\left\{f \in L^{2}\left(0, T ; L^{2}(-1,1)\right):\left\|\theta^{-3 / 2} e^{s \alpha} f\right\|_{L^{2}\left(0, T ; L^{2}(-1,1)\right)} \leqslant r\right\} .
$$

We construct a map $\mathcal{N}: \mathfrak{B}_{r} \times(-r, r) \times \mathfrak{F}_{r} \longrightarrow \mathfrak{F}_{r}$ by setting, for $y_{0} \in \mathfrak{B}_{r}, h_{0} \in(-r, r)$ and $f \in \mathfrak{F}_{r}$,

$$
\mathcal{N}\left(y_{0}, h_{0}, f\right)=\mathcal{N}(y, h),
$$

where $(y, h)$ is the controlled trajectory provided by Theorem 3.1.

Proof of Theorem 4.1. We split the proof in 3 steps.

Step 1. For each $y_{0} \in \mathfrak{B}_{r}$ and $h_{0} \in(-r, r)$, the application $\mathcal{N}\left(y_{0}, h_{0}, \cdot\right)$ maps $\mathfrak{F}_{r}$ to itself whenever $r>0$ is small enough. Indeed, by Lemma 4.1 and Lemma 3.2

$$
\begin{aligned}
& \left\|\theta^{-3 / 2} e^{s \alpha} \mathcal{N}\left(y_{0}, h_{0}, f\right)\right\|_{L^{2}\left(0, T ; L^{2}(-1,1)\right)} \leqslant C_{1}\|y\|_{\mathcal{Y}}^{2} \\
& \quad \leqslant C_{1} C_{2}^{2}\left(\left\|y_{0}\right\|_{H^{1}(-1,1)}+\left|h_{0}\right|+\left\|\theta^{-3 / 2} e^{s \alpha} f\right\|_{L^{2}\left(0, T ; L^{2}(-1,1)\right)}\right)^{2} \leqslant \frac{r}{2}
\end{aligned}
$$

whenever $r \leqslant \frac{1}{18 C_{1} C_{2}^{2}}$ (where $C_{1}>0$ is the constant from Lemma 4.1 and $C_{2}>0$ the constant from Lemma 3.2).

Step 2. For each $y_{0} \in \mathfrak{B}_{r}$ and $h_{0} \in(-r, r)$ with $r>0$ small enough, the application $\mathcal{N}\left(y_{0}, h_{0}, \cdot\right)$ is a contraction on $\mathfrak{F}_{r}$ with a uniform constant $<1$. This follows by estimating similarly as in Lemma 4.1 and Step 1, and closely follows the estimates in [26].

Step 3. Thanks to the Banach fixed point theorem, given $r>0$ small enough, for any $y_{0} \in \mathfrak{B}_{r}$ and $h_{0} \in(-r, r)$, the application $\mathcal{N}\left(y_{0}, h_{0}, \cdot\right)$ admits a unique fixed point $f \in \mathfrak{F}_{r}$, and consequently a unique solution to the control problem for (4.1).

We may thus conclude the proof of Theorem 1.1. 
Proof of Theorem 1.1. The result follows by virtue of the transformations performed in Section 2 and Theorem 4.1. Indeed, given initial data $\left(v_{0}, \ell_{0}\right) \in H^{1}\left(0, \ell_{0}\right) \times \mathbb{R}_{+}^{*}$, we consider $y_{0}(\cdot):=v_{0}\left(\ell_{0} \cdot\right)-\bar{v}$ and $h_{0}=\ell_{0}-\ell_{*}$. As $y_{0} \in H^{1}(0,1)$, we may extend it to a function $\widetilde{y}_{0} \in H^{1}(-1,1)$, which coincides with $y_{0}$ on $(0,1)$. Let $\omega=\left(\gamma_{1}, \gamma_{2}\right) \subset$ $(-1,0)$ be a non-empty set. By Theorem 4.1, there exists $r>0$ such that whenever $\left\|\widetilde{y}_{0}\right\|_{H^{1}(-1,1)}+\left|h_{0}\right| \leqslant r$, there exists a control $\widetilde{u} \in L^{2}\left(0, T ; L^{2}(\omega)\right)$ such that the solution $(y, h)$ to $(4.1)$ satisfies $y(T, \cdot)=0$ in $(-1,1)$ and $h(T)=0$. This in turn implies that the control $u(t):=y(t, 0)+\bar{v}$ guarantees the null-controllability of the boundary control system $(2.3)$ on $(0,1)$, with initial data $\left(y_{0}, h_{0}\right)$. We now set $w(t, x):=y(t, x)+\bar{v}$ in $[0, T] \times[0,1]$ and $\ell(t)=h(t)+\bar{\ell}(t)$ in $[0, T]$. It is readily seen that $(w, \ell)$ satisfy (2.2) for initial data $\left(v\left(\ell_{0} \cdot\right), \ell_{0}\right)$, as well as $w(T, \cdot)=\bar{v}$ in $(0,1)$ and $\ell(T)=\bar{\ell}(T)$. As the result is local, one also has $\ell(t)>0$ in $[0, T]$ by continuity, and thus reversing the transformation (2.1) gives the desired result.

\section{Concluding Remarks}

In this work, we addressed the local controllability of both components of the state of a one-dimensional free boundary problem governed by the viscous Burgers equation. By means of a control actuating along the fixed boundary, we showed that we may steer the fluid to constant velocity and also control the position of its free surface, whenever the difference between the initial velocities and the interface positions respectively is small enough. While the existence of this non-trivial trajectory is a particularity of the system under consideration, our result also implies its null-controllability.

We present hereinafter several topics closely related to our work.

5.1. Controllability to arbitrary trajectories. A challenging problem to which we have not given a solution herein is the controllability to arbitrary smooth trajectories for parabolic free boundary problems. Up to the best of our knowledge, this problem has not been addressed in the literature, even in the one-dimensional case. Let us give a brief overview of the issues that may arise in doing so for system (1.1).

We recall that as per Section 2, after fixing the domain for (1.1), we consider perturbations around a given smooth solution $(\bar{w}, \bar{\ell})$ of $(2.2)$ - we write $w=\bar{w}+y$ and $\ell=\bar{\ell}+h$, and keep all the terms which are linear with respect to $(y, h)$. The linearized system reads

$$
\begin{cases}y_{t}-a y_{x x}+b y_{x}+c y+d h^{\prime}+e h=0 & \text { in }(0, T) \times(0,1) \\ y(t, 0)=u(t)-\bar{u}(t), \quad y_{x}(t, 1)=0 & \text { in }(0, T) \\ h^{\prime}(t)=y(t, 1) & \text { in }(0, T) \\ y(0, x)=y_{0}(x), \quad h(0)=h_{0} & \text { in }(0,1),\end{cases}
$$

where $\bar{u}(t)=\bar{w}(t, 0), y_{0}(\cdot)=w_{0}(\cdot)-\bar{w}(0, \cdot), h_{0}=\ell_{0}-\bar{\ell}(0)$, with $a$ as in (2.4), and the remaining coefficients given by

$$
\begin{aligned}
b(t, x) & =\frac{\bar{w}(t, x)-\bar{\ell}^{\prime}(t) x}{\bar{\ell}(t)}, & c(t, x) & =\frac{\bar{w}_{x}(t, x)}{\bar{\ell}(t)} \\
d(t, x) & =-\frac{x \bar{w}_{x}(t, x)}{\bar{\ell}(t)}, & e(t, x) & =\frac{2 \bar{w}_{t}(t, x)}{\bar{\ell}(t)}+\frac{\bar{w}(t, x) \bar{w}_{x}(t, x)-x \bar{\ell}^{\prime}(t) \bar{w}_{x}(t, x)}{\bar{\ell}(t)^{2}},
\end{aligned}
$$


in $[0, T] \times[0,1]$. We remark that by applying a Banach fixed-point argument to the source term $d h^{\prime}+e h$, it can be shown that the linearized problem (5.1) is well-posed in the energy space $X_{T}=L^{2}\left(0, T ; H^{1}(-1,1)\right) \cap C^{0}\left([0, T] ; L^{2}(-1,1)\right)$.

Contrary to the specific case we treated in this paper, there is no reason as to why the factors $d, e$ would vanish for an arbitrary trajectory $(\bar{v}, \bar{\ell})$, so the finite-dimensional constraint techniques presented herein are not applicable. Thus, as done in Section 2, let us first consider a distributed control system in the extended domain $(-1,1)$ :

$$
\begin{cases}y_{t}-a y_{x x}+b y_{x}+c y+d h^{\prime}+e h=u \mathbf{1}_{\omega} & \text { in }(0, T) \times(-1,1) \\ y(t,-1)=y_{x}(t, 1)=0 & \text { in }(0, T) \\ h^{\prime}(t)=y(t, 1) & \text { in }(0, T) \\ y(0, x)=y_{0}(x), \quad h(0)=h_{0} & \text { in }(-1,1),\end{cases}
$$

where the coefficients and initial data are extended accordingly. The localized control $u=u(t, x)$ actuates inside some open, non-empty set $\omega \subsetneq(-1,0)$. Since we consider the case $d, e \not \equiv 0$, the PDE and ODE components remain coupled. Moreover the adjoint problem one obtains is more difficult to handle - multiplying (5.2) by a pair of smooth functions $(\zeta, s)$ and integrating leads us to

$$
\begin{cases}-\zeta_{t}-a \zeta_{x x}-(b \zeta)_{x}+c \zeta=0 & \text { in }(0, T) \times(-1,1) \\ \zeta(t,-1)=0, \quad \zeta_{x}(t, 1)=-\int_{-1}^{1} d \zeta \mathrm{d} x+s(t) & \text { in }(0, T) \\ s^{\prime}(t)=\int_{-1}^{1} d \zeta \mathrm{d} x & \text { in }(0, T) \\ \zeta(T, x)=\zeta_{T}(x), \quad s(T)=s_{T} & \text { in }(-1,1) .\end{cases}
$$

The adjoint problem (5.3) is much like the forward problem appearing in certain works on population dynamics, see [28] for instance. The authors prove an observability inequality for (5.2), which in our case is the forward problem. Up to the best of our knowledge, an observability inequality for (5.3) has not been shown in the literature.

Another possible strategy for tackling the null-controllability of (5.2) is to "absorb" the nonlocal terms $d h^{\prime}$ and $e h$ in the source term $f$. The fact that these terms are linear would raise an issue in proving the invariance of the fixed-point map (Step 1 in Proof of Theorem 4.1). An idea which is used in several papers on the controllability to trajectories for the non-homogeneous Navier-Stokes equations (see [12] and the references therein) is to keep the Carleman constants $s, \lambda \geqslant 1$ arbitrary throughout the proofs. Thus, when proving the fixed-point, one may appeal to these constants as an additional degree of freedom which could render the linear terms small. The main issue in applying this strategy is the compactness-uniqueness method used to prove the improved observability inequality in Proposition 3.1. Indeed, the indirect nature of this proof means that the explicit dependence of the new observability constant on the parameters $s, \lambda$ is a priori unknown. Hence, taking $s, \lambda$ arbitrarily large a posteriori may not be feasible.

5.2. Global results. As discussed in Remark 1.1, Theorem 1.1 is a local result, as while the PDE component may possess a dissipative mechanism, the asymptotic position of the free boundary is generally not known for problems of this nature. This is in part due to the lack of conservation properties satisfied by the position of the free 
boundary $\ell$, making its asymptotic position significantly more difficult to determine when compared to similar problems with a stronger coupling and set on the whole line $[33,25]$. In fact, by means of some maximum principle argument, it could be possible that the free boundary increases as time grows, which could in turn stipulate an asymptotic behavior of the velocity $v$ to a self-similar profile of the form $\frac{1}{\sqrt{t}} f\left(\frac{x}{\sqrt{t}}\right)$, well known in the context of the viscous Burgers equation set on $\mathbb{R}$ (see e.g. [36]). Thus, even the set of attraction points of trajectories of (1.1) is not evident.

It would most certainly be interesting to know whether one may prove a global controllability result in large time. This question is in fact also open in the simpler case of the one-phase Stefan problem (1.4), and also in the fluid-structure problem (1.5).

5.3. Multi-dimensional problem. One may also consider an appropriate controllability problem for the incompressible Navier-Stokes equations with a free surface, as encountered in the works of Beale [2,3]. This would represent a natural extension of our work to the multi-dimensional setting.

The main difference with the one-dimensional case presented herein and existing works on multi-dimensional fluid-rigid body control (see e.g. [20,4]) is the fact that the free boundary would be given by the graph of a space-dependent function, whence the second component of the system would be governed by an infinite-dimensional ODE and controlling this component would not represent a finite-dimensional constraint. This is an obvious impediment to the direct application of the techniques presented herein. The null-controllability of the PDE component in the two-dimensional Stefan problem in a radial geometry has been addressed in [8], following the strategy of the one-dimensional counterpart presented in [17]. However, up to the best of our knowledge, the controllability of both components in such a geometrical setting has not been addressed in the literature.

Acknowledgments. B.G. is grateful to Debayan Maity for many fruitful discussions. Both authors acknowledge the anonymous reviewers whose remarks have improved the overall quality of this work.

Funding: This project has received funding from the European Union's Horizon 2020 research and innovation programme under the Marie Sklodowska-Curie grant agreement No.765579-ConFlex. E.Z. has received funding from the Alexander von Humboldt-Professorship program, the European Research Council (ERC) under the European Union's Horizon 2020 research and innovation programme (grant agreement NO. 694126-DyCon), the Transregio 154 Project "Mathematical Modelling, Simulation and Optimization Using the Example of Gas Networks" of the German DFG, grant MTM201792996-C2-1-R COSNET of MINECO (Spain) and by the Air Force Office of Scientific Research (AFOSR) under Award NO. FA9550-18-1-0242.

\section{REFERENCES}

[1] Badra, M., And Takahashi, T. Feedback stabilization of a simplified 1d fluid-particle system. Annales de l'Institut Henri Poincaré (C) Non Linear Analysis 31, 2 (2014), 369-389.

[2] Beale, J. The initial value problem for the Navier-Stokes equations with a free surface. Comm. Pure Appl. Math. 34 (1981), 359-392.

[3] Beale, J. Large-time regularity of viscous surface waves. Arch. Ration. Mech. Anal. 84 (1984), 307-352.

[4] Boulakia, M., And Guerrero, S. Local null controllability of a fluid-solid interaction problem in dimension 3. J. Eur. Math. Soc 15 (2013), 825-856. 
[5] Caboussat, A. Numerical simulation of two-phase free surface flows. Arch. Comput. Methods Eng. 12, 2 (2005), 165-224.

[6] Caboussat, A., and Rappaz, J. Analysis of a one-dimensional free boundary flow problem. Numer. Math. 101 (2005), 67-86.

[7] Cindea, N., Micu, S., Roventa, I., and Tucsnak, M. Particle supported control of a fluidparticle system. J. Math. Pures Appl. 104, 2 (2014), 311-353.

[8] Demarque, R., and Fernández-CAra, E. Local null controllability of one-phase Stefan problems in 2d star-shaped domains. J. Evol. Equ. 18, 1 (2018), 245-261.

[9] Dunbar, W. B., Petit, N., Rouchon, P., and Martin, P. Boundary control of a nonlinear Stefan problem. In 42nd IEEE International Conference on Decision and Control (IEEE Cat. No. 03CH37475) (2003), vol. 2, IEEE, pp. 1309-1314.

[10] Dunbar, W. B., Petit, N., Rouchon, P., And Martin, P. Motion planning for a nonlinear Stefan problem. ESAIM Control Optim. Calc. Var. 9 (2003), 275-296.

[11] Ervedoza, S. Control issues and linear projection constraints on the control and on the controlled trajectory. North-W. Eur. J. of Math. 6 (2020), 165-197.

[12] Ervedoza, S., ANd SAvel, M. Local boundary controllability to trajectories for the 1d compressible Navier Stokes equations. ESAIM Control Optim. Calc. Var. 24, 1 (2018), 211-235.

[13] Fernández-Cara, E., And de Sousa, I. T. Local null controllability of a free-boundary problem for the semilinear 1d heat equation. Bull. Braz. Math. Soc. 48 (2017), 303-315.

[14] Fernández-Cara, E., And Doubova, A. Some control results for simplified one-dimensional models of fluid-solid interaction. Math. Models Methods Appl. Sci 15, 5 (2005), 783-824.

[15] Fernández-Cara, E., Guerrero, S., Imanuvilov, O., and Puel, J.-P. Local exact controllability of the Navier-Stokes system. J. Math. Pures Appl. 83, 12 (2004), 1501-1542.

[16] Fernández Cara, E., Hernández, F., and Limaco Ferrel, J. Local null controllability of a 1d Stefan problem. Bull. Braz. Math. Soc. (2018), 1-25.

[17] Fernández-Cara, E., Limaco Ferrel, J., and Dias Bezerra de Menezes, S. On the controllability of a free-boundary problem for the 1d heat equation. Syst. Cont. Lett. 87 (2016).

[18] Furskikov, A., And Imanuvilov, O. Y. Controllability of evolution equations. vol. 34 of Lecture Notes Series. Soul National University Research Institute of Mathematics Global Analysis Research Center, Seoul, 1996.

[19] Geshrovski, B. Null-controllability of perturbed porous medium gas flow. ESAIM Control Optim. Calc. Var. 26 (2020), 85.

[20] Imanuvilov, O., and Takahashi, T. Exact controllability of a fluid-rigid body system. $J$. Math. Pur. Appl. 87 (2007), 408-437.

[21] Koga, S., Diagne, M., And Krstic, M. Output feedback control of the one-phase stefan problem. In 2016 IEEE 55th Conference on Decision and Control (CDC) (2016), IEEE, pp. 526531.

[22] Koga, S., Diagne, M., And Krstic, M. Control and state estimation of the one-phase Stefan problem via backstepping design. IEEE Trans. Automat. Control 64, 2 (2018), 510-525.

[23] Koga, S., Diagne, M., Tang, S., and Krstic, M. Backstepping control of the one-phase stefan problem. In 2016 American Control Conference (ACC) (2016), IEEE, pp. 2548-2553.

[24] Koga, S., And Krstic, M. Single-boundary control of the two-phase Stefan system. Syst. Cont. Lett. 135 (2020), 104573.

[25] KoIKe, K. Long-time behavior of a point mass in a one-dimensional viscous compressible fluid and pointwise estimates of solutions. J. Differ. Eq. 271 (2021), 356-413.

[26] Liu, Y., Takahashi, T., and Tucsnak, M. Single input controllability of a simplified fluidstructure interaction model. ESAIM Control Optim. Calc. Var. 19, 1 (2013), 20-42.

[27] Maity, D., And Tucsnak, M. A maximal regularity approach to the analysis of some particulate flows. In Particles in flows, Adv. Math. Fluid Mech. Birkhäuser/Springer, 2017, pp. 1-75.

[28] Maity, D., Tucsnak, M., And Zuazua, E. Controllability and positivity constraints in population dynamics with age structuring and diffusion. J. Math. Pures Appl. 129 (2019), 153-179.

[29] Maronnier, V., Picasso, M., and Rappaz, J. Numerical simulation of free surface flows. $J$. Comput. Phys. 155, 2 (1999), 439-455.

[30] Nakoulima, O. Contrôlabilité à zéro avec contraintes sur le contrôle. CR Math. 339, 6 (2004), 405-410.

[31] Phan, D., And Rodrigues, S. Stabilization to trajectories for parabolic equations. Math. Control Signals Systems 30, 2 (2018), 11. 
[32] Ramaswamy, M., Roy, A., and Takahashi, T. Remark on the global null controllability for a viscous Burgers-particle system with particle supported control. Appl. Math. Lett. (2020), 106483.

[33] VÁzquez, J., AND Zuazua, E. Large time behavior for a simplified 1d model of fluid-solid interaction. Commun. Part. Diff. Eq. 28, 9-10 (2003), 1705-1738.

[34] Vázquez, J., And Zuazua, E. Lack of collision in a simplified 1d model of fluid-solid interaction. Math. Models Methods Appl. Sci 16, 5 (2006), 637-678.

[35] Ziane, T. A., Ouzzane, H., And Zair, O. A Carleman estimate for the two dimensional heat equation with mixed boundary conditions. C. R. Math. Acad. Sci. Paris 351, 3-4 (2013), 97-100.

[36] ZuazuA, E. Asymptotic behavior of scalar convection-diffusion equations. arXiv preprint arXiv:2003.11834 (2020).

\section{Borjan Geshkovski}

Departamento de Matemáticas

Universidad Autónoma de Madrid

28049 Madrid, Spain

$$
\text { and }
$$

Chair of Computational Mathematics

Fundación Deusto

Av. de las Universidades, 24

48007 Bilbao, Basque Country, Spain

Email address: borjan.geshkovski@uam.es

\section{Enrique Zuazua}

Chair in Applied Analysis, Alexander von Humboldt-Professorship

Department of Mathematics

Friedrich-Alexander-Universität Erlangen-Nürnberg

91058 Erlangen, Germany

$$
\text { and }
$$

Chair of Computational Mathematics

Fundación Deusto

Av. de las Universidades, 24

48007 Bilbao, Basque Country, Spain

$$
\text { and }
$$

Departamento de Matemáticas

Universidad Autónoma de Madrid

28049 Madrid, Spain

Email address: enrique.zuazua@fau.de 